\title{
Morphometric Evaluation of Posterior Funiculus Nerve Fibers in Relation to Aging
}

\author{
By \\ Shin-ichi AYABE ${ }^{\mathrm{a}, \mathrm{b}}$, Noboru GOTO ${ }^{\mathrm{a}}$, Takashi ATSUMI ${ }^{\mathrm{b}}$, Jun GOTO ${ }^{\mathrm{a}}$ \\ and Jun SUZUKI ${ }^{\mathrm{a}, \mathrm{b}}$

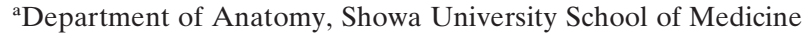 \\ ${ }^{b}$ Department of Orthopedics, Showa University Fujigaoka Hospital \\ - Received for Publication, January 17, $2005-$ \\ Key Words: Morphometry, Axon, Aging process, Luxol fast blue-periodic acid-Schiff-hematoxylin (LPH), spinal cord \\ Summary: We morphologically evaluated the size of axons in the posterior funiculus in different age groups and ex- \\ amined the changes due to aging. In the past, such studies have been conducted at the cervical spinal cord (C6) level, \\ and a decrease in the size and number of axons due to aging has been noted. The current study was conducted at the
} lower lumber spinal cord (L2) level.

Change in nerve fibers with aging is one of the major concerns in recent morphological and physiological research. A decrease in the number and area of axons in the posterior funiculus of the cervical spinal cord with aging has been proven ${ }^{1)}$. However, no such study has yet been conducted at lumber level. The difference between the two is that at the cervical cord level, the posterior funiculus is divided into two by the posterior median septum. It is further divided into fasciculus gracilis and fasciculus cuneatus by the posterior intermedial sulcus. But there is no such division at lumber cord level. The aim of this study is to find if there are changes in morphometric data related to the aging process in the axons of the lumber posterior funiculus.

\section{Material and Methods}

Research was conducted on spinal cords removed from 11 dissection cadavers $(5$ men and 6 women) ranging in age from 47 to 90 years (average age: 71 years). The causes of death were unrelated to diseases of the central nervous system. The same method as reported previously by the authors $^{2-8)}$ was used for the preparation and mea- surement of the spinal cord from dissections (fixation, washing, dehydration, embedding, staining, and morphometry). Spinal cord L2 level was identified according to the criteria published by Goto ${ }^{2)}$. The spinal segment were sliced to make transverse sections at $20 \mu \mathrm{m}$ thickness. This was followed by staining with the Luxol fast blue-periodic acidSchiff-hematoxylin (LPH) method. As shown in Fig. 1, we divided both sides of the spinal cord at L2 into medial and lateral parts, and 3 sites from each, i.e. a total of 6 areas, were selected for measurement. The right and left sides of the posterior funiculus of the same specimens were used as sampling sites. Measurement was performed on a total of 22 samples. Using an eyepiece with a square grid and a high-magnification microscope $(\times 3,500)$ equipped with an oil-immersion lens, we measured the number and cross-sectional area of myelinated axons in 6 sampling areas of $0.0030 \mathrm{~mm}^{2}$ each. The imageanalyzer consisted of (a) a microscope (BH2, Olympus, Tokyo, Japan) equipped with a drawing tube (BH2-DA, Olympus, Tokyo, Japan); (b) a digitizer (Crystizer a KC 3300, Graphic, Yokohama, Japan); and (c) a computer (NEC, Tokyo, Japan) with analyzing system software (original programming) for storage of data on-line, calculation and statistical analysis. A regression analysis

Correspondence: Noboru Goto, M.D., Professor and Chairman, Department of Anatomy, Showa University School of Medicine, 5-8, Hatanodai 1, Shinagawa-ku, Tokyo 142-8555, Japan. e-mail: goto@med.showa-u.ac.jp 


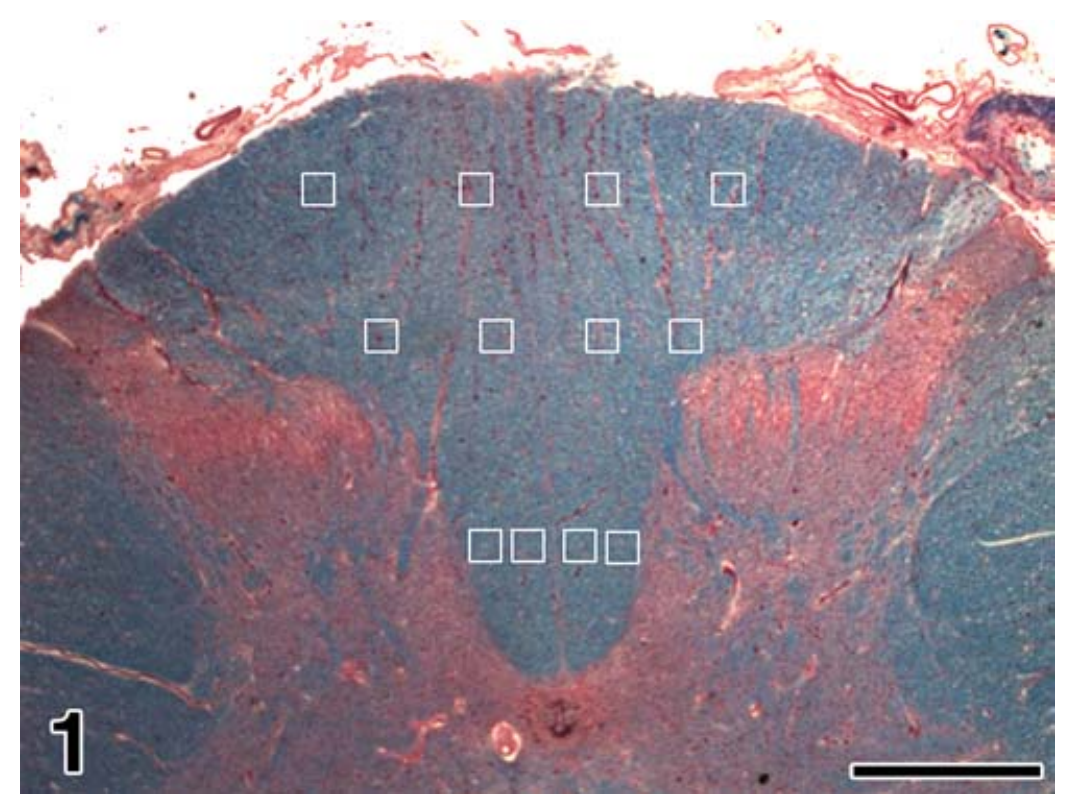

Fig. 1. Sampling area in human posterior funiculus at $\mathrm{L} 2$, bar $=1 \mathrm{~mm}$.

Table 1. Morphometric data

\begin{tabular}{|c|c|c|c|c|c|}
\hline Age group & $\mathrm{N}$ & $\begin{array}{c}\text { Number of } \\
\text { axons per } \\
\text { section } \\
\left(/ 0.018 \mathrm{~mm}^{2}\right)\end{array}$ & $\begin{array}{c}\text { Average } \\
\text { axonal area } \\
\left(\mu \mathrm{m}^{2}\right)\end{array}$ & $\begin{array}{c}\text { Total axonal } \\
\text { areas per } \\
\text { section } \\
\left(\mu \mathrm{m}^{2} / 0.018 \mathrm{~mm}^{2}\right)\end{array}$ & $\begin{array}{c}\text { Average } \\
\text { perimeter } \\
(\mu \mathrm{m})\end{array}$ \\
\hline A $(40-49)$ & 2 & $134.0 \pm 1.00$ & $3.20 \pm 0.006$ & $429.1 \pm 2.33$ & $6.25 \pm 0.06$ \\
\hline $\mathrm{B}(50-59)$ & 4 & $129.2 \pm 0.62$ & $3.13 \pm 0.032$ & $404.9 \pm 3.08$ & $6.04 \pm 0.06$ \\
\hline $\mathrm{C}(60-69)$ & 4 & $107.7 \pm 5.69$ & $2.72 \pm 0.140$ & $295.4 \pm 30.5$ & $5.71 \pm 0.10$ \\
\hline $\mathrm{D}(70-79)$ & 6 & $91.33 \pm 4.83$ & $2.65 \pm 0.092$ & $244.2 \pm 21.5$ & $5.52 \pm 0.05$ \\
\hline E (80-89) & 4 & $93.25 \pm 8.75$ & $2.63 \pm 0.110$ & $247.8 \pm 32.5$ & $5.59 \pm 0.03$ \\
\hline F (90-99) & 2 & $77.50 \pm 3.50$ & $2.63 \pm 0.085$ & $204.4 \pm 15.8$ & $5.46 \pm 0.04$ \\
\hline
\end{tabular}

was performed between age and each of the number of fibers, axonal area and axonal perimeter.

\section{Results}

Quantitative measurements for the number, cross-sectional area and perimeter of myelinated axons from respective age groups are listed in Table 1. Most of the nerve fibers in the posterior funiculus of the spinal cord were consisted of myelinated fibers. A small number of unmyelinated fibers can also be seen as shown in Fig. 2 .

\section{Number of axons}

The number of myelinated axons in an area of $0.018 \mathrm{~mm}^{2}$ (total of the 6 sampling areas) in the posterior funiculus was 104.1 (ranging from 74 to
135) on average. A statistical analysis was performed for each age group using the one-way ANOVA. The number of axons for groups D (7079 years), E (80-90 years), and F (90-100 years) was significantly smaller than that for groups A (40-49 years) and B $(50-59$ years $)(p<0.001)$. A scatter diagram and regression analysis between number and age is shown in Fig. 3. It indicates a decrease in number with aging $(\mathrm{r}=-0.86$, $\mathrm{p}<0.001$, Fig. 3).

\section{Size of axons}

The average cross-sectional area of myelinated axons in the posterior funiculus was $2.79 \mu \mathrm{m}^{2}$ (ranging from 2.40 to $3.23 \mu \mathrm{m}^{2}$ ). The average total cross-sectional area for the six sampling areas was $296.6 \mu \mathrm{m}^{2}$ (ranging from 183.9 to $431.4 \mu \mathrm{m}^{2}$ ) per unit area of $0.0030-\mathrm{mm}^{2}$. One-way ANOVA and 


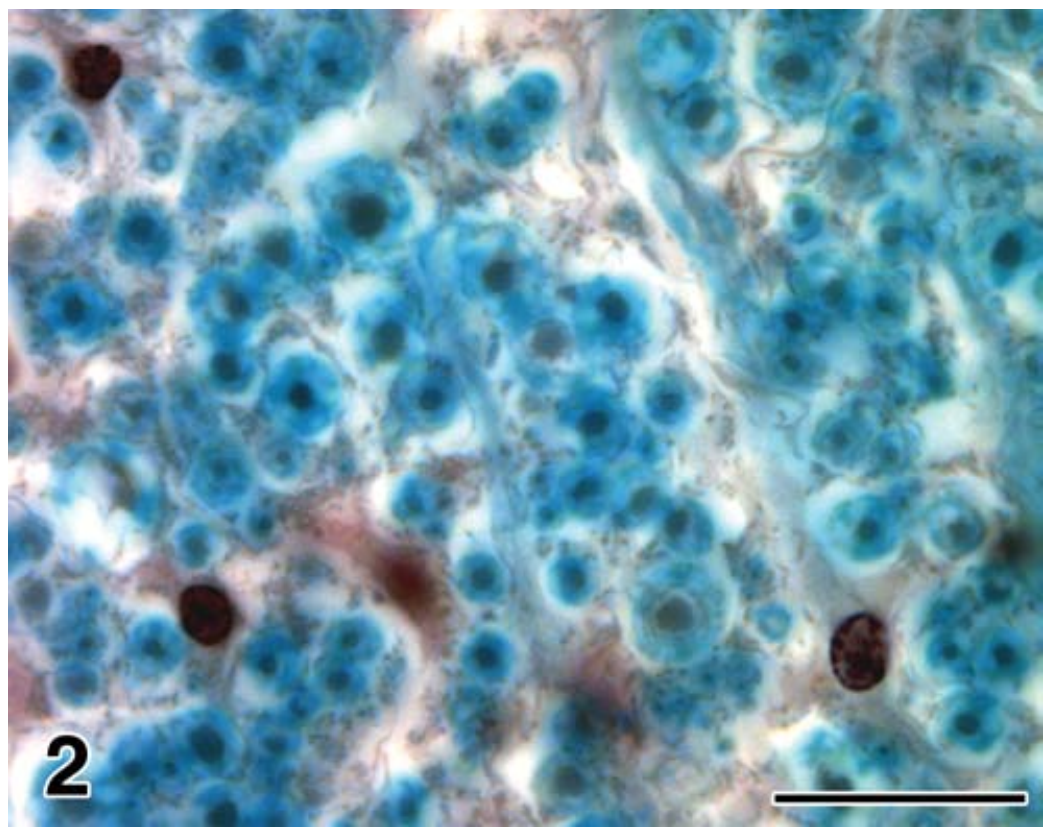

Fig. 2. Photograph of myelinated nerve fiber in human posterior funiculus, bar $=20 \mu \mathrm{m}$.

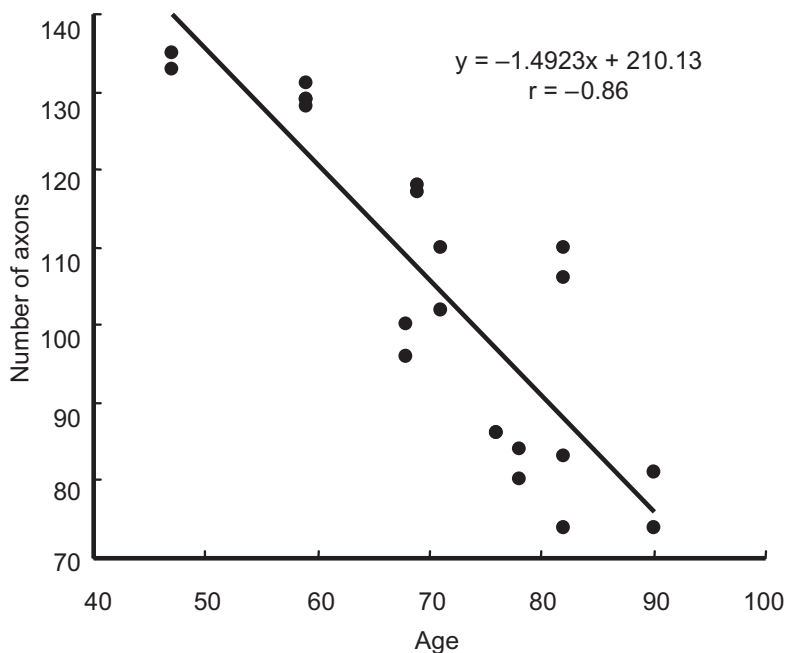

Fig. 3. Linear decrease in the number of spinal nerves in a posterior funiculus region of $0.018 \mathrm{~mm}^{2}$ with aging $(\mathrm{p}<0.001)$

regression analysis were performed. The decrease in the area size was larger in groups D (70-79), E (80-90), and F (90-100) than in groups A (40-49) and $\mathrm{B}(50-59)$ (significant difference at $\mathrm{p}<0.001$ ), as in the case of the number of axons. A scatter diagram and regression analysis between crosssectional areas (average and total) and age are shown in Figs. 4 and 5. These figures indicate a de-

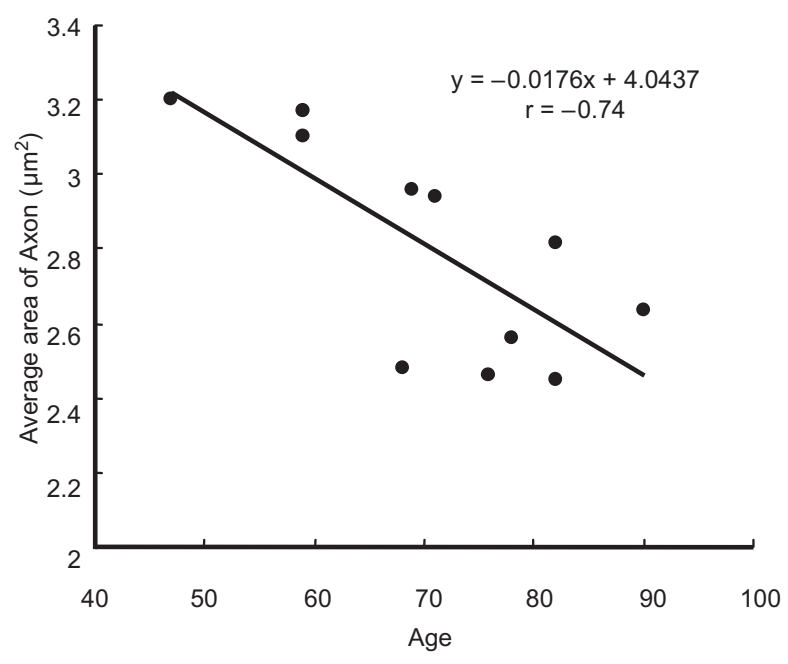

Fig. 4. Scatter diagram and regression line for the correlation between aging and average axonal area at the L2 level of the posterior funiculus $(\mathrm{p}<0.001)$.

crease in cross-sectional area with aging $(\mathrm{r}=-0.74$, $\mathrm{p}<0.001$, Fig. $4 ; \mathrm{r}=-0.85, \mathrm{p}<0.001$, Fig. 5).

\section{Perimeter of axons}

The average axonal perimeter in the spinal cord was $5.72 \mu \mathrm{m}$ (ranging from 5.35 to $6.32 \mu \mathrm{m}$ ). A significant decrease with age was noted for this as well $(r=-0.87, p<0.001$, Fig. 6). 


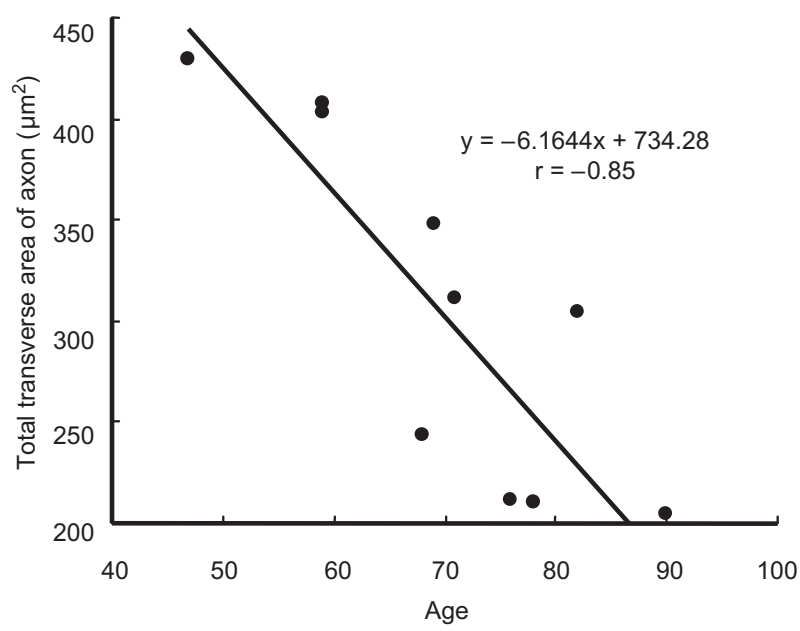

Fig. 5. Scatter diagram and regression line for the correlation between aging and total axonal area at the L2 level of the posterior funiculus $(\mathrm{p}<0.001)$.

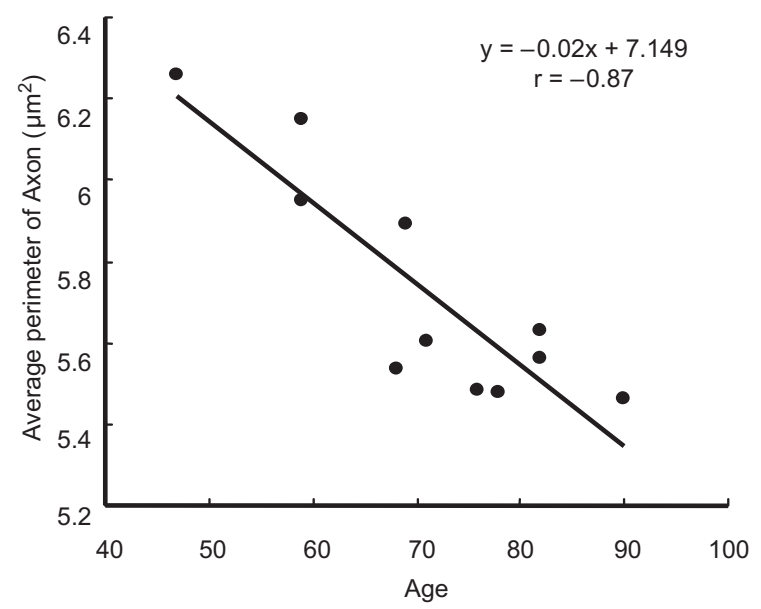

Fig. 6. Scatter diagram and regression line for the correlation between again and the axonal perimeter at the L2 level of the posterior funiculus $(\mathrm{p}<0.001)$.

\section{Discussion}

The posterior funiculus is known to be a conducting pathway for discriminative tactile, vibratory, joint position and motion sensation. Poviton ${ }^{9)}$ clinically examined various neurological changes at ages from the $20 \mathrm{~s}$ to the $80 \mathrm{~s}$, and found that most of them were connected with the aging process. Those affecting vibratory sensation, in particular, were markedly apparent.

Up to now, there have been few reports on the morphological changes in human spinal nerve fibers caused by aging. A morphological comparison of individual age groups at the cervical cord level conducted on myelinated axonal fibers in the posterior funiculus has shown a decrease in number, area, and perimeter of myelinated neuronal axons duaring the aging process ${ }^{1}$. The posterior funiculus is divided into two by posterior median septum at cervical and thoracic cord levels, and is further devided into the fasciculus gracilis and fasciculus cuneatus. This is not seen at the lumbar cord level. This study adopted a similar method to the one used in our previous reports ${ }^{1)}$ at the lumbar cord level. A significant age-related linear correlation was observed for all parameters: number, average area, total area, and average perimeter of myelinated axons within the sampling area. In other words, this proves that the aging process affects not only the cervical level but the lumber cord level as well. We may presume that the rest of the spinal cord (thoracic and sacral level) is affected in same way.

A similar method to the one used in this study could be applied to research on degeneration of the posterior funiculus, especially the funiculus gracilis, which affects fairly large proportion of elderly people.

\section{References}

1) Zhang $\mathrm{C}$, Goto $\mathrm{N}$ and Zhou $\mathrm{M}$. Morphometrics analysis and aging process of nerve fibers in the human spinal posterior funiculus. Okajimas Folia Anat Jpn 1995; 72:259-264.

2) Goto N. Gray and white matters of the spinal cord: Diagnostic criteria of the levels. Spine and Spinal Cord 1988; 62:329-333 (in Japanese).

3) Goto J, Goto N, Ezure H, Nonaka N and Ma X. Morphogial differentiation of nerve fibers: Central, peripheral, myelinated and unmyelinated. Okajimas Folia Anat Jpn 2001; 77:211-216.

4) Goto N. Discriminative staining methods for the nervous system: Luxol fast blue-periodic acid-Schiff-hematoxylin triple stain and subsidiary staining methods. Stain Technol 1987; 62:305-315.

5) Fujii $\mathrm{M}$ and Goto N. Nerve fiber analysis of the facial nerve. Ann Otol Rhinol Laryngol 1989; 98:732-735.

6) Fujii M, Goto N, Setoyama S, Okada A and Kikuchi K. Evidence for the tapering of nerve axons. Showa Univ $\mathrm{J}$ Med Sci 1994; 6:179-184.

7) Yanagisawa K, Goto N, Kimura T and Tanaka J. Myelinated nerve fiber analysis and aging process of the deep peroneal nerve in man. Showa Univ J Med 1994; 54:249-254 (in Japanese).

8) Tang W, Goto N, Tanaka J and Otsuka N. Myalinated nerve fiber analysis of the human small splanchnic nerve. Okajimas Folia Anat Jpn 1997; 74:93-98.

9) Potvin AR, Syndulko K, Tourtellotte WW, Lemmeon JA and Potvin JH. Human neurologic function and the aging process. J Am Geriatr Soc 1980; 28:1-9. 